\title{
Exact nonadditive kinetic potentials for embedded density functional theory
}

\author{
Jason D. Goodpaster, ${ }^{1}$ Nandini Ananth, ${ }^{1}$ Frederick R. Manby, ${ }^{2}$ and Thomas F. Miller III, \\ ${ }^{1}$ Division of Chemistry and Chemical Engineering, California Institute of Technology, \\ Pasadena, California 91125, USA \\ ${ }^{2}$ Centre for Computational Chemistry, School of Chemistry, University of Bristol, \\ Bristol BS8 ITS, United Kingdom
}

(Received 26 April 2010; accepted 12 July 2010; published online 23 August 2010)

\begin{abstract}
We describe an embedded density functional theory (DFT) protocol in which the nonadditive kinetic energy component of the embedding potential is treated exactly. At each iteration of the Kohn-Sham equations for constrained electron density, the Zhao-Morrison-Parr constrained search method for constructing Kohn-Sham orbitals is combined with the King-Handy expression for the exact kinetic potential. We use this formally exact embedding protocol to calculate ionization energies for a series of three- and four-electron atomic systems, and the results are compared to embedded DFT calculations that utilize the Thomas-Fermi (TF) and the Thomas-Fermi-von Weisacker approximations to the kinetic energy functional. These calculations illustrate the expected breakdown due to the TF approximation for the nonadditive kinetic potential, with errors of 30\%$80 \%$ in the calculated ionization energies; by contrast, the exact protocol is found to be accurate and stable. To significantly improve the convergence of the new protocol, we introduce a density-based switching function to map between the exact nonadditive kinetic potential and the TF approximation in the region of the nuclear cusp, and we demonstrate that this approximation has little effect on the accuracy of the calculated ionization energies. Finally, we describe possible extensions of the exact protocol to perform accurate embedded DFT calculations in large systems with strongly overlapping subsystem densities. (C) 2010 American Institute of Physics. [doi:10.1063/1.3474575]
\end{abstract}

\section{INTRODUCTION}

Orbital-free embedded density functional theory (e-DFT) is an appealing method for calculating the electronic structure of complex molecular systems. It provides a formally exact framework for dividing the total electronic density of a system into subsystem densities that can be separately calculated. ${ }^{1-4}$ This feature of e-DFT allows for the development of multiphysics strategies in which the electronic density for the region of central interest is calculated using high-accuracy methods, while the electronic density for surrounding regions is obtained using more coarse approximations. $^{5-7}$

However, in addition to the usual approximations for the basis set and the exchange-correlation functional that appear in Kohn-Sham (KS) DFT, ${ }^{8}$ e-DFT requires the evaluation of a nonadditive contribution to the kinetic energy from the subsystem densities. ${ }^{4}$ This term, which is typically largest for cases in which the subsystem densities are strongly overlapping, ${ }^{9}$ is a significant source of error in many e-DFT calculations, and it generally limits the method to applications in which the subsystem densities involve nonbonded or weakly interacting molecular groups. ${ }^{4,9,10}$ Although encouraging progress towards the accurate calculation of the nonadditive kinetic energy contribution have been reported, ${ }^{9,11-16}$ more work in this direction is needed.

In this paper, we present a formally exact protocol for calculating the nonadditive kinetic energy contribution in e-DFT calculations, and we report calculations in which the protocol is applied to atomic systems that exhibit strongly overlapping subsystem densities. These results suggest new methods to systematically, efficiently, and accurately perform e-DFT calculations for large systems, which we discuss.

\section{ORBITAL-FREE EMBEDDED DFT}

Suppose that the entire electronic density $\rho_{\mathrm{AB}}$ for a closed-shell system is divided into two subsystems, $\rho_{\mathrm{A}}$ and $\rho_{\mathrm{B}}$, such that $\rho_{\mathrm{AB}}=\rho_{\mathrm{A}}+\rho_{\mathrm{B}}$. The one-electron orbitals that give rise to these subsystem electronic densities obey the coupled Kohn-Sham equations for constrained electron density $^{4}$ (KSCED)

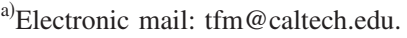




$$
\begin{aligned}
& {\left[-\frac{1}{2} \nabla^{2}+V_{\mathrm{eff}}^{\mathrm{KSCED}}\left[\rho_{\mathrm{A}}, \rho_{\mathrm{B}} ; \mathbf{r}\right]\right] \phi_{i}^{\mathrm{A}}(\mathbf{r})=\epsilon_{i}^{\mathrm{A}} \phi_{i}^{\mathrm{A}}(\mathbf{r}), \quad i=1, \ldots, \frac{N_{\mathrm{A}}}{2},} \\
& {\left[-\frac{1}{2} \nabla^{2}+V_{\mathrm{eff}}^{\mathrm{KSCED}}\left[\rho_{\mathrm{B}}, \rho_{\mathrm{A}} ; \mathbf{r}\right]\right] \phi_{i}^{\mathrm{B}}(\mathbf{r})=\epsilon_{i}^{\mathrm{B}} \phi_{i}^{\mathrm{B}}(\mathbf{r}), \quad i=1, \ldots, \frac{N_{\mathrm{B}}}{2},}
\end{aligned}
$$

where $N_{\mathrm{A}}$ and $N_{\mathrm{B}}$ are the number of electrons in the respective subsystems

$$
\begin{aligned}
& \rho_{\mathrm{A}}(\mathbf{r})=2 \sum_{i=1}^{N_{\mathrm{A}} / 2}\left|\phi_{i}^{\mathrm{A}}(\mathbf{r})\right|^{2}, \quad \text { and } \\
& \rho_{\mathrm{B}}(\mathbf{r})=2 \sum_{i=1}^{N_{\mathrm{B}} / 2}\left|\phi_{i}^{\mathrm{B}}(\mathbf{r})\right|^{2} .
\end{aligned}
$$

In these coupled equations, $V_{\text {eff }}^{\mathrm{KSCED}}\left[\rho_{\mathrm{A}}, \rho_{\mathrm{B}} ; \mathbf{r}\right]$ is the KS effective potential for subsystem A embedded in subsystem $\mathrm{B}$

$$
\begin{aligned}
V_{\mathrm{eff}}^{\mathrm{KSCD}}\left[\rho_{\mathrm{A}}, \rho_{\mathrm{B}} ; \mathbf{r}\right]= & v_{\mathrm{ne}}(\mathbf{r})+v_{\mathrm{J}}\left[\rho_{\mathrm{AB}} ; \mathbf{r}\right]+v_{\mathrm{xc}}\left[\rho_{\mathrm{AB}} ; \mathbf{r}\right] \\
& +v_{\text {nad }}\left[\rho_{\mathrm{A}}, \rho_{\mathrm{B}} ; \mathbf{r}\right],
\end{aligned}
$$

and $V_{\text {eff }}^{\mathrm{KSCED}}\left[\rho_{\mathrm{B}}, \rho_{\mathrm{A}} ; \mathbf{r}\right]$ is the similarly defined KS effective potential for subsystem B embedded in subsystem A. The contributions to the KS effective potential include

$$
\begin{aligned}
& v_{\mathrm{ne}}(\mathbf{r})=-\sum_{i}^{N_{\mathrm{nuc}}} \frac{Z_{i}}{\left|\mathbf{r}-\mathbf{R}_{i}\right|}, \\
& v_{\mathrm{J}}\left[\rho_{\mathrm{AB}} ; \mathbf{r}\right]=\int \frac{\rho_{\mathrm{AB}}\left(\mathbf{r}^{\prime}\right)}{\left|\mathbf{r}^{\prime}-\mathbf{r}\right|} d \mathbf{r}^{\prime}, \text { and } \\
& v_{\mathrm{xc}}\left[\rho_{\mathrm{AB}} ; \mathbf{r}\right]=\left[\left.\frac{\delta E_{\mathrm{xc}}[\rho]}{\delta \rho}\right|_{\rho=\rho_{\mathrm{AB}}}\right](\mathbf{r}),
\end{aligned}
$$

which are the usual nuclear-electron Coulomb potential, Hartree potential, and exchange-correlation potential, respectively, and $N_{\text {nuc }}$ is the number of nuclei in the system. The final term in $V_{\mathrm{eff}}^{\mathrm{KSCED}}\left[\rho_{\mathrm{B}}, \rho_{\mathrm{A}} ; \mathbf{r}\right]$ is the nonadditive kinetic potential (NAKP)

$$
\begin{aligned}
v_{\text {nad }}\left[\rho_{\mathrm{A}}, \rho_{\mathrm{B}} ; \mathbf{r}\right] & =\left[\frac{\delta T_{s}^{\mathrm{nad}}\left[\rho_{\mathrm{A}}, \rho_{\mathrm{B}}\right]}{\delta \rho_{\mathrm{A}}}\right](\mathbf{r}) \\
& =\left.\frac{\delta T_{S}[\rho]}{\delta \rho}\right|_{\rho=\rho_{\mathrm{AB}}}(\mathbf{r})-\left.\frac{\delta T_{s}[\rho]}{\delta \rho}\right|_{\rho=\rho_{\mathrm{A}}}(\mathbf{r}),
\end{aligned}
$$

which is obtained from the functional derivative of the nonadditive component of the noninteracting kinetic energy

$$
T_{s}^{\mathrm{nad}}\left[\rho_{\mathrm{A}}, \rho_{\mathrm{B}}\right]=T_{s}\left[\rho_{\mathrm{AB}}\right]-T_{s}\left[\rho_{\mathrm{A}}\right]-T_{s}\left[\rho_{\mathrm{B}}\right] .
$$

The total energy functional for the embedded system is

$$
\begin{aligned}
E\left[\rho_{\mathrm{AB}}\right]= & T_{s}\left[\rho_{\mathrm{A}}\right]+T_{s}\left[\rho_{\mathrm{B}}\right]+T_{s}^{\mathrm{nad}}\left[\rho_{\mathrm{A}}, \rho_{\mathrm{B}}\right]+V_{\mathrm{ne}}\left[\rho_{\mathrm{AB}}\right] \\
& +J\left[\rho_{\mathrm{AB}}\right]+E_{\mathrm{xc}}\left[\rho_{\mathrm{AB}}\right],
\end{aligned}
$$

where the last three terms on the right hand side (RHS) are the nuclear-electron Coulomb energy, Hartree energy, and exchange-correlation energy.

Two aspects of the orbital-free embedding DFT formulation are worth emphasizing. First, like conventional KS-DFT, it is a theory that is exact in principle, but practical calculations must employ an approximate form for the unknown exchange-correlation functional. Second, unlike conventional KS-DFT calculations, the embedding formulation introduces a NAKP because the KS orbitals for subsystem A are not necessarily orthogonal to those of subsystem B. Without knowledge of the exact functional for the noninteracting kinetic energy, this creates a second source of approximation in the e-DFT approach. The significance of the NAKP is system dependent, with the most severe cases including those for which the subsystem densities $\rho_{\mathrm{A}}$ and $\rho_{\mathrm{B}}$ greatly overlap. $^{4,9,17,18}$

The noninteracting kinetic energy for the density corresponding to a set of $\mathcal{N}$ closed-shell orbitals is

$$
T_{s}[\rho]=2 \sum_{i=1}^{\mathcal{N}}\left\langle\phi_{i}\left|-\frac{1}{2} \nabla^{2}\right| \phi_{i}\right\rangle
$$

Standard approximations to this kinetic energy functional include the Thomas-Fermi (TF) result for the homogenous electron gas $^{19,20}$

$$
T_{\mathrm{TF}}[\rho]=C_{\mathrm{TF}} \int \rho^{5 / 3}(\mathbf{r}) d \mathbf{r},
$$

where $C_{\mathrm{TF}}=\frac{3}{10}\left(3 \pi^{2}\right)^{2 / 3}$, and the von Weizsäcker $(\mathrm{vW})$ result for the limit of a one-electron density ${ }^{21}$

$$
T_{\mathrm{vW}}[\rho]=\frac{1}{8} \int \frac{|\nabla \rho(\mathbf{r})|^{2}}{\rho(\mathbf{r})} d \mathbf{r} .
$$

Other approximate kinetic energy functionals can be constructed using the strategies from the development of exchange-correlation functionals. For example, the PW91k kinetic energy functional ${ }^{11,12}$ employs the analytical form of the Perdew-Wang (PW91) exchange functional, ${ }^{22}$ and the TW02 functional ${ }^{14}$ and the PBE2, PBE3, and PBE4 functionals ${ }^{9}$ utilize the form suggested by Becke. ${ }^{23}$ These functionals have been shown to successfully describe weakly interacting systems and coordination compounds. ${ }^{9}$ Furthermore, King and Handy ${ }^{24}$ have employed the exact relationship between KS orbitals and the kinetic potential to systematically parameterize approximate kinetic energy functionals, 
with encouraging results. And kinetic energy functionals developed using linear response corrections to the homogeneous electron gas have been shown to work well for metals. ${ }^{15,25,26}$ However, no approximate kinetic energy functional has been demonstrated to yield accurate results for embedded subsystems that are connected by covalent bonds. ${ }^{9,18,27}$

\section{THE EXACT NONADDITIVE KINETIC POTENTIAL}

For each iteration of the KSCED equations [Eqs. (1) and (2)], $\left\{\phi_{i}^{\mathrm{A}}\right\}$ and $\left\{\phi_{i}^{\mathrm{B}}\right\}$ (and thus $\rho_{\mathrm{A}}$ and $\rho_{\mathrm{B}}$ ) are known from either the previous iteration or the initial guess, and the NAKP must be calculated. We employ a two-step protocol to obtain the exact NAKP. In the first step, a Levy constrained search $^{28}$ (LCS) or equivalent method is used to determine the full set of orthogonal KS orbitals, $\left\{\phi_{i}^{\mathrm{AB}}\right\}$, that correspond to the total density $\rho_{\mathrm{AB}}$. In the second step, the NAKP is calculated from the orbital sets $\left\{\phi_{i}^{\mathrm{AB}}\right\},\left\{\phi_{i}^{\mathrm{A}}\right\}$, and $\left\{\phi_{i}^{\mathrm{B}}\right\}$.

\section{A. Step 1: The LCS}

Given a total electron density $\rho_{\mathrm{AB}}$, the fully orthogonal KS orbitals $\left\{\phi_{i}^{\mathrm{AB}}\right\}$ can be calculated from a LCS, in which the noninteracting kinetic energy is minimized with respect to one-electron orbitals that are constrained to yield $\rho_{\mathrm{AB}}{ }^{28}$ Alternatively, we employ the approach of Zhao, Morrison, and Parr (ZMP), ${ }^{29-31}$ in which the full set of KS orbitals are obtained by solving the one-electron equations

$$
\left[-\frac{1}{2} \nabla^{2}-\sum_{i}^{N_{\text {nuc }}} \frac{Z_{i}}{\left|\mathbf{r}-\mathbf{R}_{i}\right|}+V_{c}^{\lambda}(\mathbf{r})\right] \phi_{i}^{\mathrm{AB}, \lambda}(\mathbf{r})=\epsilon_{i} \phi_{i}^{\mathrm{AB}, \lambda}(\mathbf{r}), \quad i=1, \ldots, \frac{N_{\mathrm{AB}}}{2},
$$

where $N_{\mathrm{AB}}=N_{\mathrm{A}}+N_{\mathrm{B}}$,

$$
V_{c}^{\lambda}(\mathbf{r})=\lambda \int \frac{\rho_{\mathrm{AB}}\left(\mathbf{r}^{\prime}\right)-\tilde{\rho}_{\mathrm{AB}}\left(\mathbf{r}^{\prime}\right)}{\left|\mathbf{r}^{\prime}-\mathbf{r}\right|} d \mathbf{r}^{\prime},
$$

$\tilde{\rho}_{\mathrm{AB}}(\mathbf{r})=2 \sum_{i=1}^{N_{\mathrm{AB}} / 2}\left|\phi_{i}^{\mathrm{AB}}(\mathbf{r})\right|^{2}$, and $V_{c}^{\lambda}(\mathbf{r})$ is a potential energy function that restrains the $\tilde{\rho}_{\mathrm{AB}}(\mathbf{r})$ to the target density $\rho_{\mathrm{AB}}(\mathbf{r})$. Solution of Eq. (15) in the limit $\lambda \rightarrow \infty$ is equivalent to performing the LCS. $^{29-31}$

In practice, Eq. (15) is solved for six large, but finite, values of $\lambda$, and the KS orbitals and eigenvalues are obtained via extrapolation. ${ }^{29-31}$ For each value of $\lambda$, the $\left\{\epsilon_{i}^{\lambda}\right\},\left\{\phi_{i}^{\mathrm{AB}, \lambda}\right\}$, and $\left\{\nabla^{2} \phi_{i}^{\mathrm{AB}, \lambda}\right\}$ are calculated and stored on a spatial grid. For the orbitals, extrapolation to $\lambda \rightarrow \infty$ is performed via expansion to third order in $(1 / \lambda)$,

$\phi_{i}^{\mathrm{AB}, \lambda}(\mathbf{r})=\phi_{i}^{\mathrm{AB}}(\mathbf{r})+\frac{1}{\lambda} a_{i}^{(1)}(\mathbf{r})+\frac{1}{\lambda^{2}} a_{i}^{(2)}(\mathbf{r})+\frac{1}{\lambda^{3}} a_{i}^{(3)}(\mathbf{r})$,

with a linear least-squares fit of the expansion coefficients $\left\{\phi_{i}^{\mathrm{AB}}(\mathbf{r}), a_{i}^{(1)}(\mathbf{r}), a_{i}^{(2)}(\mathbf{r}), a_{i}^{(3)}(\mathbf{r})\right\}$ at each value of $\mathbf{r}$. The $\left\{\nabla^{2} \phi_{i}^{\mathrm{AB}}\right\}$ are similarly obtained via extrapolation at each value of $\mathbf{r}$, while each $\epsilon_{i}$ requires only a single extrapolation. With the $\left\{\phi_{i}^{\mathrm{AB}}\right\}$ and $\left\{\nabla^{2} \phi_{i}^{\mathrm{AB}}\right\}$ obtained on the spatial grid, the noninteracting kinetic energy for the total system can be calculated via numerical integration using Eq. (12).

\section{B. Step 2: Exact kinetic potentials from KS orbitals}

To calculate the NAKP from the orbital sets $\left\{\phi_{i}^{\mathrm{AB}}\right\},\left\{\phi_{i}^{\mathrm{A}}\right\}$, and $\left\{\phi_{i}^{\mathrm{B}}\right\}$, we extend the approach developed by King and Handy. $^{32}$

Minimization of the electronic energy with respect to the total electron density $\rho_{\mathrm{AB}}$ yields the stationary condition ${ }^{8}$

$$
\left.\frac{\delta T_{s}[\rho]}{\delta \rho}\right|_{\rho=\rho_{\mathrm{AB}}}(\mathbf{r})+v_{\mathrm{ne}}(\mathbf{r})+v_{\mathrm{J}}\left[\rho_{\mathrm{AB}} ; \mathbf{r}\right]+v_{\mathrm{xc}}\left[\rho_{\mathrm{AB}} ; \mathbf{r}\right]=\mu_{\mathrm{AB}},
$$

where $\mu_{\mathrm{AB}}$ is a Lagrange multiplier that imposes the constraint $\int \rho_{\mathrm{AB}}(\mathbf{r}) d \mathbf{r}=N_{\mathrm{AB}}$. Furthermore, rearrangement of the usual KS equations yields

$$
\frac{2}{\rho_{\mathrm{AB}}(\mathbf{r})} \sum_{i}^{N_{\mathrm{AB}} / 2}\left(-\frac{1}{2} \phi_{i}^{\mathrm{AB}}(\mathbf{r}) \nabla^{2} \phi_{i}^{\mathrm{AB}}(\mathbf{r})-\epsilon_{i} \phi_{i}^{\mathrm{AB}}(\mathbf{r})^{2}\right)+v_{\mathrm{ne}}(\mathbf{r})+v_{\mathrm{J}}\left[\rho_{\mathrm{AB}} ; \mathbf{r}\right]+v_{\mathrm{xc}}\left[\rho_{\mathrm{AB}} ; \mathbf{r}\right]=0 .
$$

Comparison of these two results leads to an exact expression for the total kinetic potential ${ }^{32}$

$$
\left.\frac{\delta T_{s}[\rho]}{\delta \rho}\right|_{\rho=\rho_{\mathrm{AB}}}(\mathbf{r})=\frac{2}{\rho_{\mathrm{AB}}(\mathbf{r})} \sum_{i}^{N_{\mathrm{AB}} / 2}\left(-\frac{1}{2} \phi_{i}^{\mathrm{AB}}(\mathbf{r}) \nabla^{2} \phi_{i}^{\mathrm{AB}}(\mathbf{r})-\epsilon_{i} \phi_{i}^{\mathrm{AB}}(\mathbf{r})^{2}\right)+\mu_{\mathrm{AB}} .
$$

Analogous results can be derived for each of the embedded subsystems. Specifically, the electron density for subsystem A also obeys a stationary condition ${ }^{2}$ 


$$
\left.\frac{\delta T_{s}[\rho]}{\delta \rho}\right|_{\rho=\rho_{\mathrm{A}}}(\mathbf{r})+v_{\mathrm{ne}}(\mathbf{r})+v_{\mathrm{J}}\left[\rho_{\mathrm{AB}} ; \mathbf{r}\right]+v_{\mathrm{xc}}\left[\rho_{\mathrm{AB}} ; \mathbf{r}\right]+v_{\mathrm{nad}}\left[\rho_{\mathrm{A}}, \rho_{\mathrm{B}} ; \mathbf{r}\right]=\mu_{\mathrm{A}},
$$

where $\mu_{\mathrm{A}}$ is the Lagrange multiplier that imposes the constraint $\int \rho_{\mathrm{A}}(\mathbf{r}) d \mathbf{r}=N_{\mathrm{A}}$. Combination of Eq. (21) with Eq. (1) results in an exact expression for the subsystem kinetic potential

$$
\left.\frac{\delta T_{s}[\rho]}{\delta \rho}\right|_{\rho=\rho_{\mathrm{A}}}(\mathbf{r})=\frac{2}{\rho_{\mathrm{A}}(\mathbf{r})} \sum_{i}^{N_{\mathrm{A}} / 2}\left(-\frac{1}{2} \phi_{i}^{\mathrm{A}}(\mathbf{r}) \nabla^{2} \phi_{i}^{\mathrm{A}}(\mathbf{r})-\epsilon_{i}^{\mathrm{A}} \phi_{i}^{\mathrm{A}}(\mathbf{r})^{2}\right)+\mu_{\mathrm{A}}
$$

which can be compared with kinetic potential for the total system in Eq. (20).

Insertion of Eqs. (18) and (21) into Eq. (9) yields $\mu_{\mathrm{AB}}=\mu_{\mathrm{A}}$, and since $\mathrm{A}$ is an arbitrarily chosen subsystem, we likewise obtain $\mu_{\mathrm{AB}}=\mu_{\mathrm{B}}$, or $\mu_{\mathrm{A}}=\mu_{\mathrm{B}}$. This result has a simple physical interpretation. In the zero temperature limit, the Lagrange multipliers $\mu_{\mathrm{A}}$ and $\mu_{\mathrm{B}}$, are equivalent to the chemical potential for the subsystem electronic densities. ${ }^{8}$ Solution to the KSCED equations thus yields densities that are in equilibrium with respect to the number of electrons in each subsystem.

Finally, insertion of Eqs. (20) and (22) into Eq. (9) yields the desired expression for the NAKP

$$
\begin{aligned}
v_{\text {nad }}\left[\rho_{\mathrm{A}}, \rho_{\mathrm{B}} ; \mathbf{r}\right] & \\
= & \frac{2}{\rho_{\mathrm{AB}}(\mathbf{r})} \sum_{i}^{N_{\mathrm{AB}} / 2}\left(-\frac{1}{2} \phi_{i}^{\mathrm{AB}}(\mathbf{r}) \nabla^{2} \phi_{i}^{\mathrm{AB}}(\mathbf{r})-\epsilon_{i} \phi_{i}^{\mathrm{AB}}(\mathbf{r})^{2}\right) \\
& -\frac{2}{\rho_{\mathrm{A}}(\mathbf{r})} \sum_{i}^{N_{\mathrm{A}} / 2}\left(-\frac{1}{2} \phi_{i}^{\mathrm{A}}(\mathbf{r}) \nabla^{2} \phi_{i}^{\mathrm{A}}(\mathbf{r})-\epsilon_{i}^{\mathrm{A}} \phi_{i}^{\mathrm{A}}(\mathbf{r})^{2}\right) .
\end{aligned}
$$

Note that the ZMP protocol generally yields a constant shift in the calculated set of KS eigenenergies, $\left\{\epsilon_{i}^{\lambda}\right\},{ }^{29}$ in Eq. (23), we see that this leads only to a constant shift in $v_{\text {nad }}\left[\rho_{\mathrm{A}}, \rho_{\mathrm{B}} ; \mathbf{r}\right]$ and causes no change in any calculated observables. Throughout this study, the NAKP is shifted such that it approaches zero at large distances.

Previous work has observed that the NAKP can be expressed in terms of the stationary condition for the total system [Eq. (18)] and a subsystem [Eq. (21)], ${ }^{33,34}$ and Visscher et $a l .{ }^{35}$ have developed an e-DFT strategy in which the total electronic density from a KS-DFT calculation is used to test the accuracy of approximate kinetic energy functionals. However, the approach presented here allows for the calculation of the total electronic density using e-DFT, without introducing approximations for the NAKP. It is straightforward to show that Eq. (23) recovers the limit for weakly overlapping subsystem densities that is reported in Ref. 33 .

In another approach that does not utilize the exact framework of the KSCED equations, Aguado and co-workers ${ }^{36,37}$ employ an embedding strategy in which a potential inversion method such as ZMP is used to restrain the sum of subsystem densities to a total system density. In its refined version, ${ }^{37}$ this approach allows for the simultaneous determination of the electronic density partition and the embedding potential, and it has been pursued as a strategy for including local electron correlation into large systems. However, this method also requires the input of the total electron density from a Hartree-Fock or KS-DFT calculation on the full system.

Other e-DFT strategies also express the kinetic potential in terms of the KS orbitals, as we have done here. For example, Huang and Carter $^{38}$ report an explicit expression for the kinetic potential in terms of the KS orbitals, using the assumption that the noninteracting kinetic energy is a linear functional of the density; an empirical parameter is included in their result to account for nonlinear effects. The approach presented here involves no adjustable parameters and no assumptions about the linearity of the kinetic energy functional.

\section{Computational details}

Calculations are performed on four atomic systems: $\mathrm{Li}$, $\mathrm{Ne}^{7+}, \mathrm{Q}_{2.5}^{-0.5}$, and $\mathrm{Be}$, where $\mathrm{Q}_{2.5}^{-0.5}$ is a model three-electron atom that has a nuclear charge of +2.5 . In all e-DFT calculations, we take $\rho_{\mathrm{A}}$ to be the density for a single $2 s$ electron, and $\rho_{\mathrm{B}}$ includes all other electrons. The KSCED equations for each system were solved with $\rho_{\mathrm{B}}$ fixed at the density obtained from the corresponding orbitals of an unrestricted KS-DFT calculation on the full system; this is justified for the cases studied here because solution of the KSCED equations for $\rho_{\mathrm{A}}$ subject to a fixed $\rho_{\mathrm{B}} \leq \rho_{0}$ (at all $\mathbf{r}$ ), where $\rho_{0}$ is the exact ground state density for the full system, ensures the exact calculation of the ground state energy and ground state density. ${ }^{4}$ All calculations were performed using in-house codes, and all results are reported in atomic units.

\section{Basis sets}

All calculations were performed using the fully uncontracted cc-pVTZ basis set of Gaussian-type orbitals (GTOs), ${ }^{39}$ with only the $s$-type orbitals included. For calculations on $Q_{2.5}^{-0.5}$, the $\mathrm{Li}$ basis set was used. For $\mathrm{Ne}^{7+}$, the most diffuse $s$-orbital was removed to facilitate convergence. Although not presented, all calculations were also repeated with Slater-type orbitals (STOs), which led to somewhat improved convergence but very similar numerical accuracy.

\section{DFT implementation details}

For all applications considered here, $\rho_{\mathrm{A}}$ is an open shell system, and the calculations were performed using the unrestricted KS formalism. Prior studies have compared the relative merits of using restricted versus unrestricted open-shell $\mathrm{KS}-\mathrm{DFT},{ }^{40}$ but we note that there is no theoretical obstacle to formulating a restricted $\mathrm{KS}$ version of our embedding protocol. Details for the unrestricted KSCED equations are given 


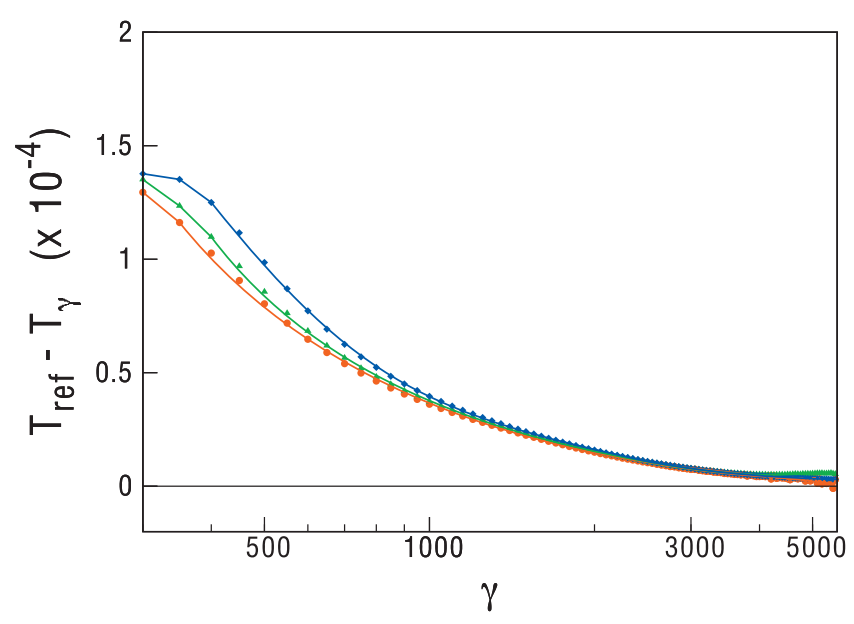

FIG. 1. The difference between the noninteracting kinetic energy $T_{\mathrm{s}}[\rho]$ from KS-DFT and from the ZMP method, plotted as a function of $\gamma$. The extrapolation is performed using $\{\lambda\}=\{\gamma-j \tau\}, j=5,4, \ldots, 0$, and using $\tau$ of 10 (red), 20 (green), and 40 (blue). See text for details. Energies are reported in atomic units.

in the Appendix. All calculations employ the Slater exchange functional $^{41}$ and the Vosko, Wilk, and Nusair correlation functional. ${ }^{42}$ In calculating $V_{\mathrm{eff}}^{\mathrm{KSCED}}\left[\rho_{\mathrm{A}}, \rho_{\mathrm{B}} ; \mathbf{r}\right]$, a uniform radial grid is used to evaluate the exchange-correlation potential, $\left\{\phi_{i}^{\mathrm{AB}}\right\},\left\{\nabla^{2} \phi_{i}^{\mathrm{AB}}\right\}$, and the NAKP. Upon convergence of the KSCED equations, the same radial grid is used to evaluate the exchange-correlation energy and to numerically integrate the kinetic energy. For $\mathrm{Be}$ and $\mathrm{Li}$ the grid extends from $r=0$ to 15 , while for $Q_{2.5}^{-0.5}, r=0$ to 20 and $\mathrm{Ne}^{7+}, r=0$ to 2 . For $\mathrm{Be}, \mathrm{Li}$, and $Q$ the grid density is 2000 points $/ a_{0}$ and for $\mathrm{Ne}^{7+}, 20000$ points $/ a_{0}$. We note that future applications that employ either a nonuniform ${ }^{43}$ or variational $^{44}$ mesh will require fewer grid-points to achieve the same level of accuracy. Unless otherwise stated, the iterative solution of the KSCED equations was deemed converged when the total energy of the system changed by less than $10^{-8}$ hartree between successive iterations.

\section{ZMP extrapolation}

To examine the extrapolation error associated with the ZMP method, convergence tests were performed for the case of the $\mathrm{Li}$ atom. The total density for the system, $\rho_{\mathrm{AB}}$, and the reference value for the non-interacting kinetic energy were calculated from a full KS calculation. This $\rho_{\mathrm{AB}}$ was used to define the restraint potential [Eq. (16)], and the ZMP extrapolation was performed using six equally spaced values of $\lambda$ (i.e., $\{\lambda\}=\{\gamma-j \tau\}$, where $j=5,4, \ldots, 0)$. For a given pair of parameters $\gamma$ and $\tau$, the noninteracting kinetic energy was numerically integrated, and the extrapolation error was taken to be the difference between this result and the reference value from the full KS calculation. Figure 1 presents this calculated error as a function $\gamma$ and for various values of $\tau$. These results indicate that the extrapolation error decreases to within $0.1 \mathrm{mH}$ for $\gamma>500$, and the spacing parameter $\tau$ has only a small effect. The error decreases to within $1 \mu \mathrm{H}$ for larger values of $\gamma$. Results reported hereafter employ $\gamma$
$=600$ and $\tau=10$. The orbitals from Eq. (15) were deemed converged when all occupied orbital coefficients changed less than $10^{-7}$ between successive iterations.

The ZMP extrapolation scheme used here does not constrain the normalization of the orbitals. In general, we found that extrapolation violated normalization by less then $0.01 \%$, and it was found that normalizing the orbitals after extrapolation led to less than $0.1 \mathrm{mH}$ change in the total energy. The results reported here do not include a posteriori orbital normalization.

\section{RESULTS}

e-DFT was performed for a series of three-electron systems, $Q_{2.5}^{-0.5}, \mathrm{Li}$, and $\mathrm{Ne}^{7+}$, as well as the four-electron $\mathrm{Be}$ atom. For each application, $\rho_{\mathrm{A}}$ was chosen to include a single $2 s$ electron, and the remaining electrons were included in $\rho_{\mathrm{B}}$. In addition to using the exact embedding protocol described here, the NAKP in the embedding calculations was treated using the approximate TF kinetic energy functional $\left[T_{\mathrm{TF}}[\rho]\right.$, Eq. (13)] and the TFvW functional with the standard 1/9 mixing parameter $\left(T_{\mathrm{TF}}[\rho]+\frac{1}{9} T_{\mathrm{vW}}[\rho]\right)$. It has been previously demonstrated that local and semi-local kinetic energy functionals fail to reproduce atomic shell structure, ${ }^{45-47}$ so these applications present a problematic scenario for the approximate TF and TFvW functionals and a significant challenge for the new embedding protocol.

Figure 2 presents the $\rho_{\mathrm{A}}$ obtained in these e-DFT calculations. For reference, Fig. 2 also includes the $2 s$ orbital density from the full KS-DFT calculation. Absolute agreement between the KS-DFT results and the e-DFT results would only be expected if all results were obtained with the exact exchange-correlation functional. Nonetheless, since all calculations in this study employ the same approximate exchange-correlation functional, comparison of the e-DFT and KS-DFT results tests the accuracy of the various NAKP descriptions.

Figure 2 clearly demonstrates the sensitivity of e-DFT calculations to the method of treating the NAKP. In comparison to KS-DFT, the e-DFT results from the approximate TF and TFvW functionals are peaked at significantly shorter radial distances, and they qualitatively fail to capture the nodal structure. The vW correction to the TF functional actually worsens the agreement with the KS-DFT reference. The exact embedding protocol describe here, however, is graphically indistinguishable from the KS-DFT result.

Further evaluation of the e-DFT methods can be obtained by comparing the calculated one-electron ionization energies (IEs) for the various methods. The e-DFT IE is calculated from the difference between the total electron energy from Eq. (11) and the energy from a full KS-DFT calculation performed on the ionized ( $N-1$ electron) system. These results are presented in Table I, which again illustrates the qualitative shortcomings of the approximate NAKP treatments. For the approximate NAKP descriptions, the relative error between the e-DFT result and the KS-DFT result for the IEs ranges from $30 \%$ to $60 \%$ for three-electron systems, and up to $80 \%$ for Be. As has been observed previously, ${ }^{48}$ including the $\mathrm{vW}$ gradient correction decreases the accuracy 

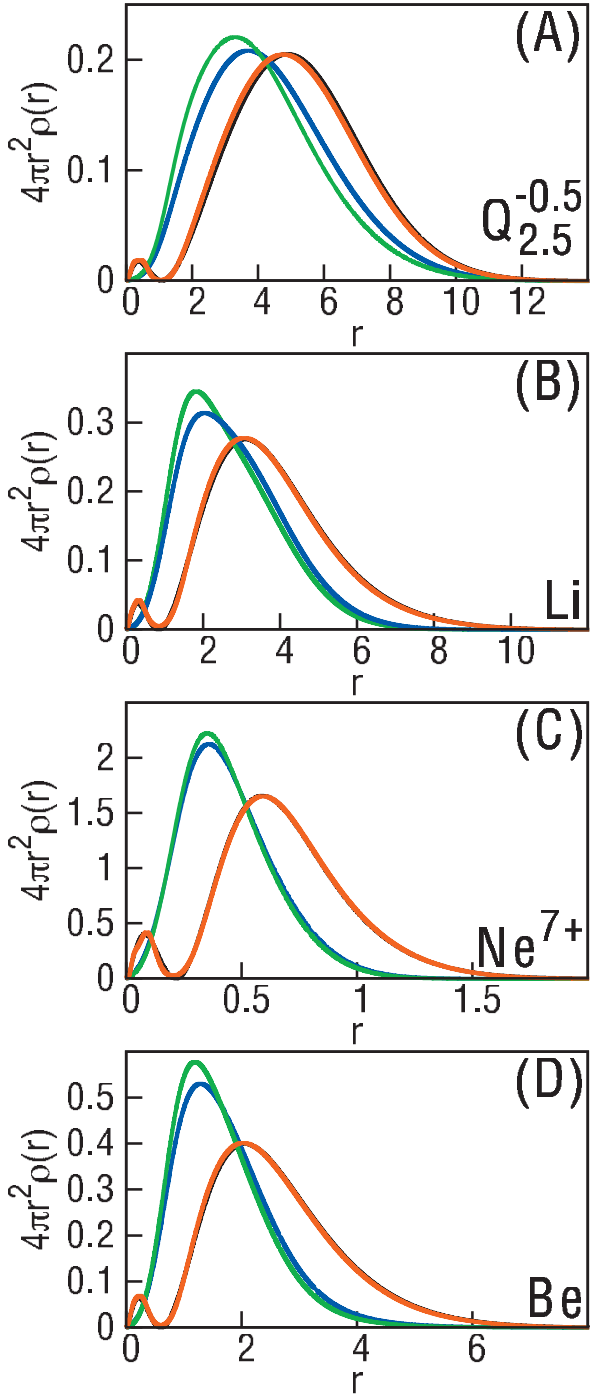

FIG. 2. The $2 s$ electron density $\left(\rho_{\mathrm{A}}\right)$ for (A) the $Q_{2.5}^{-0.5}$ ion, (B) the Li atom, (C) the $\mathrm{Ne}^{+7}$ ion, and (D) the Be atom. Calculations performed using e-DFT with the nonadditive kinetic energy calculated using our exact protocol (red), the TF functional (blue), and the TFvW functional (green). The black curve, which is nearly indistinguishable from the exact protocol, presents the results from the full KS-DFT calculation. All quantities are reported in atomic units.

of the IE calculation. The exact embedding protocol almost completely eliminates these differences with the reference calculation, with errors of less than $0.2 \%$ for $Q_{2.5}^{-0.5}, \mathrm{Li}$, and $\mathrm{Be}$ and with an error of less $4 \%$ for $\mathrm{Ne}^{7+}$.

The lower accuracy of our embedding protocol for the case of $\mathrm{Ne}^{7+}$ arises from the description of the nuclear cusp. The KSCED equations converged slowly for this case, and the convergence threshold had to be raised to $10^{-5}$ hartrees. By changing from GTOs to STOs (results not shown), the convergence problem was removed, and it was found that for all four applications, the IEs obtained using our e-DFT protocol were within $1 \%$ of the full KS-DFT result. Below, we describe how the use of a simple switching function for the NAKP in the cusp region also removes these convergence problems for the GTOs, while preserving the accuracy of the IE calculation.

We note that the ionization of the closed shell Be atom presents an electronic structure challenge that is similar to
TABLE I. Total energy (TE) and ionization energy (IE) obtained using KS-DFT and e-DFT.

\begin{tabular}{|c|c|c|c|c|}
\hline Atom & Calculation & $\mathrm{TE}$ & IE & $\begin{array}{c}\text { Error }^{\mathrm{a}} \\
(\%)\end{array}$ \\
\hline \multicolumn{5}{|l|}{$Q_{2.5}^{-0.5}$} \\
\hline & KS & -4.799405 & 0.060142 & $\cdots$ \\
\hline & TFvW & -4.835122 & 0.095258 & 59.99 \\
\hline & $\mathrm{TF}$ & -4.819221 & 0.079357 & 33.28 \\
\hline & Exact embedding & -4.799510 & 0.060247 & 0.18 \\
\hline \multicolumn{5}{|l|}{$\mathrm{Li}$} \\
\hline & KS & -7.343870 & 0.201098 & $\cdots$ \\
\hline & TFvW & -7.443321 & 0.300549 & 49.45 \\
\hline & $\mathrm{TF}$ & -7.408509 & 0.265737 & 32.14 \\
\hline & Exact embedding ${ }^{\mathrm{b}}$ & -7.344046 & 0.201274 & 0.09 \\
\hline \multicolumn{5}{|l|}{$\mathrm{Ne}^{7+}$} \\
\hline & KS & -101.964612 & 8.754056 & $\cdots$ \\
\hline & TFvW & -106.413890 & 13.203334 & 50.83 \\
\hline & $\mathrm{TF}$ & -105.630042 & 12.419486 & 41.87 \\
\hline & Exact embedding ${ }^{c}$ & -102.294207 & 9.083650 & 3.77 \\
\hline \multicolumn{5}{|l|}{$\mathrm{Be}$} \\
\hline & KS & -14.447017 & 0.331698 & $\cdots$ \\
\hline & TFvW & -14.717243 & 0.601924 & 81.47 \\
\hline & $\mathrm{TF}$ & -14.635950 & 0.520631 & 56.96 \\
\hline & Exact embedding ${ }^{\mathrm{b}}$ & -14.447463 & 0.328900 & 0.13 \\
\hline
\end{tabular}

$\overline{\overline{\text { aPercentage error } \text { is calculated with respect to the corresponding }}}$ KS-DFT IE.

${ }^{\mathrm{b}} \mathrm{KSCED}$ equations converged to $10^{-7}$ hartree.

${ }^{c} \mathrm{KSCED}$ equations converged to $10^{-5}$ hartree.

the homolytic cleavage of a covalent bond. From the perspective of the NAKP, this atomic system is especially challenging since both electrons in the $2 s$ "bond" are colocalized on a single attractive center. The difficulty of this particular case is confirmed by the especially poor description provided by the TF and TFvW functionals for the IE of the Be atom. The excellent accuracy of the new embedding protocol for this case suggests that the method will allow for accurate e-DFT calculations in which the subsystems are linked by covalent bonds.

Figure 3 illustrates the KSCED potentials, $V_{\mathrm{eff}}^{\mathrm{KSCED}}\left[\rho_{\mathrm{A}}, \rho_{\mathrm{B}} ; \mathbf{r}\right]$, and the corresponding NAKPs, $v_{\text {nad }}\left[\rho_{\mathrm{A}}, \rho_{\mathrm{B}} ; \mathbf{r}\right]$, that are obtained from the exact embedding calculations. For each system, the similarity between these two potentials illustrates the dominance of the NAKP at short distances. However, the NAKP decays rapidly, and the KSCED potential is dominated at larger distances by the Coulombic terms [Eq. (5)]. Although it is not visible from the scale of the plots in Fig. 3, the $v_{\text {nad }}\left[\rho_{\mathrm{A}}, \rho_{\mathrm{B}} ; \mathbf{r}\right]$ term comprises less than $1 \%$ of the $V_{\text {eff }}^{\mathrm{KSCED}}\left[\rho_{\mathrm{A}}, \rho_{\mathrm{B}} ; \mathbf{r}\right]$ for distances greater than 3 a.u. for all cases. (For $\mathrm{Ne}^{7+}$, this regime is reached at 0.43 a.u.)

Comparison of the NAKPs in Figs. 3(e)-3(h) with the densities in Fig. 2 illustrates that the nodal structure in the $2 s$ electron density is enforced by the NAKP. For each system, the large outer peak in the NAKP coincides with the nodal feature in the $2 s$ density. Unlike the KS-DFT results, we note that the densities obtained using e-DFT in Fig. 2 do not exhibit a genuine radial node, since $\rho_{\mathrm{A}}$ corresponds to the ground-state eigenvector of Eq. (1). A large peak in the e-DFT effective potential is therefore essential to achieve the 

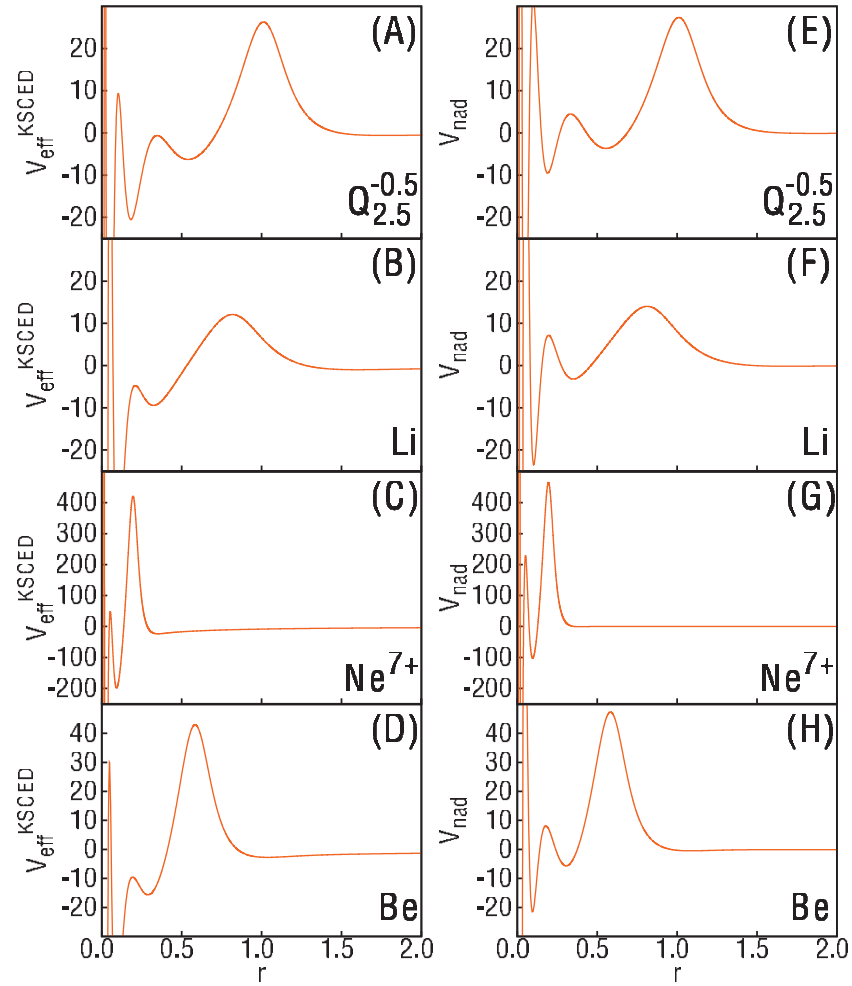

FIG. 3. The KSCED effective potential, $V_{\text {eff }}^{\mathrm{KSCED}}\left[\rho_{\mathrm{A}}, \rho_{\mathrm{B}} ; \mathbf{r}\right]$, for (a) the $Q_{2.5}^{-0.5}$ ion, (b) the $\mathrm{Li}$ atom, (c) the $\mathrm{Ne}^{+7}$ ion, and (d) the Be atom and the NAKP, $v_{\text {nad }}\left[\rho_{\mathrm{A}}, \rho_{\mathrm{B}} ; \mathbf{r}\right]$, for (e) the $Q_{2.5}^{-0.5}$ ion, (f) the $\mathrm{Li}$ atom, $(\mathrm{g})$ the $\mathrm{Ne}^{+7}$ ion, and (h) the $\mathrm{Be}$ atom using the e-DFT protocol presented here. All quantities are reported in atomic units.

correct $2 s$ shell structure. The NAKPs obtained from the approximate $\mathrm{TF}$ and $\mathrm{TFvW}$ functionals do not exhibit this pronounced peak (not shown), which leads to the poor descriptions for the 2 s electron density (Fig. 2) and the IE (Table I).

In addition to the pronounced outer-most peak for each NAKP in Figs. 3(e)-3(h), oscillations at short distances are observed. This oscillatory behavior is sensitive to the basis set representation. Small changes in the orbital coefficients for regions of low density give rise to large changes in the kinetic potential [Eq. (23)], resulting in slow convergence of the KSCED equations. (These oscillations are not observed when the density vanishes at large distances since the basis set expansion is dominated by only the slowest-decaying function in that regime.) Using STOs rather than GTOs, the NAKP oscillations at short distances were diminished (not shown), and the iterative convergence was improved. In future applications of the exact embedding protocol with GTOs, the use of the convergence acceleration algorithms such as DIIS (Ref. 49) may prove beneficial. However, we now demonstrate that the problems associated with NAKP oscillations can be alleviated with a simple modification of Eq. (23).

As $\rho_{\mathrm{A}}$ vanishes close to the nucleus, evaluation of the second term in Eq. (23) becomes unstable, leading to slow convergence of the KSCED equations. This is avoided by introducing a switching function that changes from the exact expression for the kinetic potential of subsystem A to the corresponding TF approximation near the nucleus
TABLE II. Total energy (TE) and ionization energy (IE) obtained using e-DFT with the NAKP switching function [Eq. (24)].

\begin{tabular}{lccc}
\hline \hline Atom & TE & IE & $\begin{array}{c}\text { Error }^{\mathrm{a}} \\
(\%)\end{array}$ \\
\hline$Q_{2.5}^{-0.5}$ & -4.799142 & 0.059879 & 0.44 \\
$\mathrm{Li}$ & -7.342720 & 0.199948 & 0.57 \\
$\mathrm{Ne}^{7+}$ & -101.843497 & 8.632941 & 1.38 \\
$\mathrm{Be}$ & -14.443703 & -0.328383 & 1.00 \\
\hline \hline
\end{tabular}

${ }^{\mathrm{a}}$ Percentage error is calculated with respect to the corresponding KS-DFT IE values reported in Table I.

$$
\begin{aligned}
v_{\mathrm{nad}}\left[\rho_{\mathrm{A}}, \rho_{\mathrm{B}} ; \mathbf{r}\right] & \\
= & \frac{2}{\rho_{\mathrm{AB}}(\mathbf{r})} \sum_{i=1}^{N_{\mathrm{AB}} / 2}\left(-\frac{1}{2} \phi_{i}^{\mathrm{AB}}(\mathbf{r}) \nabla^{2} \phi_{i}^{\mathrm{AB}}(\mathbf{r})-\epsilon_{i} \phi_{i}^{\mathrm{AB}}(\mathbf{r})^{2}\right) \\
& -\frac{2}{\rho_{\mathrm{A}}(\mathbf{r})} \sum_{i=1}^{N_{\mathrm{A}} / 2}\left(-\frac{1}{2} \phi_{i}^{\mathrm{A}}(\mathbf{r}) \nabla^{2} \phi_{i}^{\mathrm{A}}(\mathbf{r})-\epsilon_{i}^{\mathrm{A}} \phi_{i}^{\mathrm{A}}(\mathbf{r})^{2}\right) \\
& \times\left(1-f\left[\rho_{\mathrm{B}} ; \mathbf{r}\right]\right)-\left(\frac{5}{3} C_{\mathrm{TF}} \rho_{\mathrm{A}}^{2 / 3}\right) f\left[\rho_{\mathrm{B}} ; \mathbf{r}\right],
\end{aligned}
$$

where $f\left[\rho_{\mathrm{B}} ; \mathbf{r}\right]$ is the smooth switching function

$$
f\left[\rho_{\mathrm{B}} ; \mathbf{r}\right]=\frac{1}{e^{\kappa\left(-\rho_{\mathrm{B}}(\mathbf{r})+\rho_{\mathrm{B}}^{\prime}\right)}+1} .
$$

Previous work used a similar function to switch between approximate expressions for the NAKP in the vicinity of the nuclear cusp. ${ }^{48}$ The parameters $\rho_{\mathrm{B}}^{\prime}$ and $\kappa$ determine the radial distance and the abruptness with which switching occurs, respectively. The parameter $\rho_{\mathrm{B}}^{\prime}$ was related to the integrated electron density in the cusp region, setting $\rho_{\mathrm{B}}^{\prime}=\rho_{\mathrm{B}}\left(r^{\prime}\right)$, where

$$
\xi=4 \pi \int_{0}^{r^{\prime}} r^{2} \rho_{\mathrm{B}}(r) d r .
$$

e-DFT results obtained using range of values for $\kappa$ and $\xi$ were compared to determine robust parameters for the switching function. Setting $\kappa=50$, we varied $\xi$ over the range from 0.4 to 0.8 for $\mathrm{Li}$ and $\mathrm{Ne}^{7+}$, which led to changes in the total calculated energy of less than 0.4 and $5 \mathrm{mH}$, respectively. Similarly, setting $\xi=0.6$ and varying $\kappa$ over the range from 50 to 500 led to differences of less than $0.1 \mathrm{mH}$ for both $\mathrm{Li}$ and $\mathrm{Ne}$.

Using the NAKP expression in Eq. (24) with $\xi=0.6$ and $\kappa=50$, our e-DFT protocol was applied to all four systems, and the results are presented in Table II. All calculations reached full $10^{-8}$ convergence within 80 iterations of the KSCED equations, in contrast with the calculations using Eq. (23), which was difficult to converge in some cases even with 2000 iterations. Furthermore, the e-DFT calculations with the modified NAKP expression in Eq. (24) yields good accuracy in comparison to the full KS-DFT equations, with less than $1.5 \%$ error in the IE for all cases.

For the Li atom, Fig. 4 compares the NAKP, the KSCED effective potential, and the $2 s$ electron density obtained by solving the KSCED equations using either Eq. (23) (black) or Eq. (24) (red) for the NAKP. The black curves in this figure are the same as those for Li in Figs. 2 and 3. It is clear 

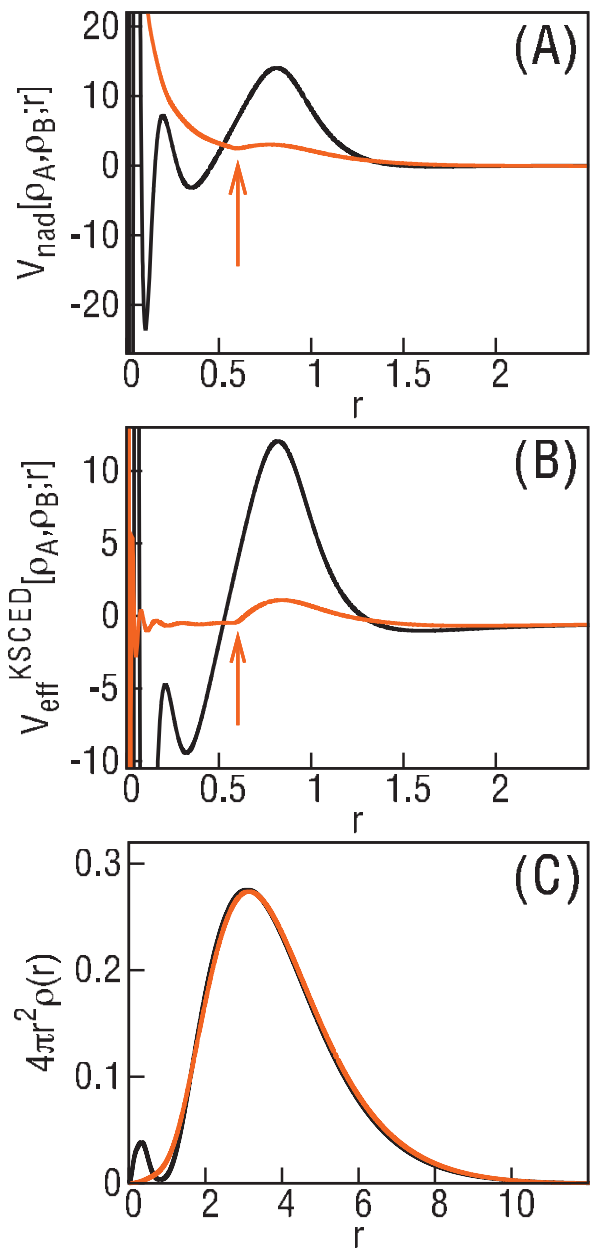

FIG. 4. (a) The NAKP, (b) the KSCED effective potential, and (c) the $2 s$ electron density $\left(\rho_{\mathrm{A}}\right)$ for the $\mathrm{Li}$ atom, obtained using exact embedding (black) and using the modified NAKP in Eq. (24) (red). The arrow indicates the radial distance at which switching occurs. All quantities are reported in atomic units.

from Fig. 4(a) that at short distances, the switching function produces a relatively featureless, repulsive NAKP due to the $\mathrm{TF}$ approximation; the arrow in this figure indicates the radial distance $r^{\prime}$ that corresponds to the parameter $\xi=0.6$. Figure 4(b) illustrates that the repulsive NAKP largely cancels the attractive electron-nuclear Coulomb term in the KSCED effective potential [Eq. (5)]. As $\rho_{\mathrm{A}}$ vanishes at the nucleus, the KSCED effective potential must also approach zero. ${ }^{33}$ The remaining oscillations at short radial distances in Fig. 4(b) are an artifact of the first term on the RHS of Eq. (24). Finally, Fig. 4(c) demonstrates that the $2 s$ electron density that is obtained using the switching function does not reproduce the features of the radial node, but it recovers the exact embedding result for distances beyond 1 a.u. This close agreement at large distances is expected ${ }^{50}$ from the accuracy of the IE calculations in Table II. In light of the much improved convergence efficiency, use of the NAKP expression in Eq. (24) compares favorably with exact embedding via Eq. (23).

\section{EXTENSION TO LARGER SYSTEMS}

The calculations reported here demonstrate a proof-ofprinciple for the exact calculation of the NAKP. However, direct application of the presented algorithm to large systems is expected to be impractical, since most previously reported applications of the ZMP extrapolation have been limited to systems with less than 15 atoms. ${ }^{36,37,51-54}$ Nonetheless, the short-ranged nature of the NAKP [see Figs. 3(e)-3(h)] suggests several strategies for employing our e-DFT protocol in larger systems.

For example, suppose that subsystem $B$ is further divided into fragments $\left(\mathrm{B}_{1}, \mathrm{~B}_{2}, \ldots, \mathrm{B}_{f}\right)$, and consider the sum of the NAKP terms due to the individual fragments ${ }^{1}$

$$
v_{\text {nad }}\left[\rho_{\mathrm{A}}, \rho_{\mathrm{B}} ; \mathbf{r}\right] \approx \sum_{i=1}^{f}\left(\left.\frac{\delta T_{s}[\rho]}{\delta \rho}\right|_{\rho=\rho_{\mathrm{A}}+\rho_{B_{i}}}-\left.\frac{\delta T_{s}[\rho]}{\delta \rho}\right|_{\rho=\rho_{\mathrm{A}}}\right) .
$$

This equation is exact in the limit of one fragment, and its implementation with our protocol will avoid ZMP extrapolation for anything larger than the union of subsystem A with a single fragment.

The assumption in Eq. (27) that the NAKP is additive among the fragments must be tested. However, any error introduced from this assumption can be partially corrected using an approximate local or semilocal kinetic energy functional

$$
\begin{aligned}
v_{\text {nad }}\left[\rho_{\mathrm{A}}, \rho_{\mathrm{B}} ; \mathbf{r}\right] & \\
\approx & \left(\left.\frac{\delta T_{s}[\rho]^{(\mathrm{appr})}}{\delta \rho}\right|_{\rho=\rho_{\mathrm{A}}+\rho_{\mathrm{B}}}-\left.\frac{\delta T_{s}[\rho]^{(\mathrm{appr})}}{\delta \rho}\right|_{\rho=\rho_{\mathrm{A}}}\right) \\
& -\sum_{i=1}^{f}\left(\left.\frac{\delta T_{s}[\rho]^{(\mathrm{appr})}}{\delta \rho}\right|_{\rho=\rho_{\mathrm{A}}+\rho_{B_{i}}}-\left.\frac{\delta T_{s}[\rho]^{(\mathrm{appr})}}{\delta \rho}\right|_{\rho=\rho_{\mathrm{A}}}\right) \\
& +\sum_{i=1}^{f}\left(\left.\frac{\delta T_{s}[\rho]^{(\mathrm{exact})}}{\delta \rho}\right|_{\rho=\rho_{\mathrm{A}}+\rho_{B_{i}}}-\left.\frac{\delta T_{s}[\rho]^{(\mathrm{exact})}}{\delta \rho}\right|_{\rho=\rho_{\mathrm{A}}}\right) .
\end{aligned}
$$

Here, the first term on the RHS corresponds to the NAKP obtained from the approximate local or semilocal kinetic energy functional for the full system. In the second term, the contribution due to each of the fragments using the approximate functional is removed, and in the third term, each of the fragment contributions is replaced using the exact protocol. The short-ranged nature of the NAKP suggests that distancebased cutoffs can be employed with summations in Eqs. (27) and (28), allowing for significant computational savings.

\section{CONCLUSIONS}

We have described a general and formally exact protocol for treating the nonadditive kinetic potential in embedded density functional theory calculations. In applications to a series of three- and four-electron atomic systems that exhibit strongly overlapping subsystem densities, we have demonstrated that the new approach is accurate and stable, despite the known failures of the approximate TF kinetic energy functional for problems of this kind. We have also shown that improved convergence of the KSCED equations can be obtained with appropriate switching of the NAKP in the vi- 
cinity of the nuclear cusps, and we have described possible strategies for the scalable implementation of our embedding protocol in large systems. Ongoing work includes implementation and testing of the new protocol for molecular systems, as well as more extensive comparison against approximate kinetic energy functionals. Natural applications of exact embedding include the rigorous calculation of one-electron pseudopotentials, the calculation of DFT embedding potentials for use with high-level $a b$ initio calculations on small subsystems, ${ }^{5,15,55,56}$ and the accurate implementation of the "molecular embedding" strategy in which each molecule of a large system is assigned to a different embedded subsystem. ${ }^{10}$

\section{ACKNOWLEDGMENTS}

This work was partially supported by a Camille and Henry Dreyfus Foundation New Faculty Award and an Alfred P. Sloan Foundation Research Fellowship (T.F.M.).

\section{APPENDIX: UNRESTRICTED OPEN-SHELL E-DFT}

For unrestricted open-shell e-DFT calculations, the density of each subsystem is further partitioned into $\alpha$ and $\beta$ spin densities, such that $\rho_{\mathrm{AB}}=\rho_{\mathrm{A}}^{\alpha}+\rho_{\mathrm{A}}^{\beta}+\rho_{\mathrm{B}}^{\alpha}+\rho_{\mathrm{B}}^{\beta}$. This leads to the KSCED equations

$$
\begin{aligned}
& {\left[-\frac{1}{2} \nabla^{2}+V_{\mathrm{eff}}^{\mathrm{KSCED}}\left[\rho_{\mathrm{A}}^{\alpha}, \rho_{\mathrm{A}}^{\beta}, \rho_{\mathrm{B}}^{\alpha}, \rho_{\mathrm{B}}^{\beta} ; \mathbf{r}\right]\right] \phi_{i}^{\mathrm{A}, \alpha}(\mathbf{r})=\epsilon_{i}^{\mathrm{A}, \alpha} \phi_{i}^{\mathrm{A}, \alpha}(\mathbf{r}), \quad i=1, \ldots, N_{\mathrm{A}}^{\alpha},} \\
& {\left[-\frac{1}{2} \nabla^{2}+V_{\mathrm{eff}}^{\mathrm{KSCED}}\left[\rho_{\mathrm{A}}^{\beta}, \rho_{\mathrm{A}}^{\alpha}, \rho_{\mathrm{B}}^{\beta}, \rho_{\mathrm{B}}^{\alpha} ; \mathbf{r}\right]\right] \phi_{i}^{\mathrm{A}, \beta}(\mathbf{r})=\epsilon_{i}^{\mathrm{A}, \beta} \phi_{i}^{\mathrm{A}, \beta}(\mathbf{r}), \quad i=1, \ldots, N_{\mathrm{A}}^{\beta},} \\
& {\left[-\frac{1}{2} \nabla^{2}+V_{\mathrm{eff}}^{\mathrm{KSCED}}\left[\rho_{\mathrm{B}}^{\alpha}, \rho_{\mathrm{B}}^{\beta}, \rho_{\mathrm{A}}^{\alpha}, \rho_{\mathrm{A}}^{\beta} ; \mathbf{r}\right]\right] \phi_{i}^{\mathrm{B}, \alpha}(\mathbf{r})=\epsilon_{i}^{\mathrm{B}, \alpha} \phi_{i}^{\mathrm{B}, \alpha}(\mathbf{r}), \quad i=1, \ldots, N_{\mathrm{B}}^{\alpha},} \\
& {\left[-\frac{1}{2} \nabla^{2}+V_{\mathrm{eff}}^{\mathrm{KSCED}}\left[\rho_{\mathrm{B}}^{\beta}, \rho_{\mathrm{B}}^{\alpha}, \rho_{\mathrm{A}}^{\beta}, \rho_{\mathrm{A}}^{\alpha} ; \mathbf{r}\right]\right] \phi_{i}^{\mathrm{B}, \beta}(\mathbf{r})=\epsilon_{i}^{\mathrm{B}, \beta} \phi_{i}^{\mathrm{B}, \beta}(\mathbf{r}), \quad i=1, \ldots, N_{\mathrm{B}}^{\beta} .}
\end{aligned}
$$

Here, $N_{\mu}^{\nu}$ is the number of electrons in each subsystem, and $\rho_{\mu}^{\nu}(\mathbf{r})=\sum_{i=1}^{N_{\mu}^{\nu}}\left|\phi_{i}^{\mu, \nu}(\mathbf{r})\right|^{2}$, where $\mu \in\{\mathrm{A}, \mathrm{B}\}$ and $\nu \in\{\alpha, \beta\}$. The KSCED effective potential, $V_{\mathrm{eff}}^{\mathrm{KSCED}}\left[\rho_{\mathrm{A}}^{\alpha}, \rho_{\mathrm{A}}^{\beta}, \rho_{\mathrm{B}}^{\alpha}, \rho_{\mathrm{B}}^{\beta} ; \mathbf{r}\right]$, is

$$
\begin{aligned}
V_{\mathrm{eff}}^{\mathrm{KSCED}} & {\left[\rho_{\mathrm{A}}^{\alpha}, \rho_{\mathrm{A}}^{\beta}, \rho_{\mathrm{B}}^{\alpha}, \rho_{\mathrm{B}}^{\beta} ; \mathbf{r}\right] } \\
= & v_{\mathrm{ne}}(\mathbf{r})+v_{\mathrm{J}}\left[\rho_{\mathrm{AB}} ; \mathbf{r}\right] \\
& +v_{\mathrm{xc}}\left[\left(\rho_{\mathrm{A}}^{\alpha}+\rho_{\mathrm{B}}^{\alpha}\right),\left(\rho_{\mathrm{A}}^{\beta}+\rho_{\mathrm{B}}^{\beta}\right) ; \mathbf{r}\right]+v_{\mathrm{nad}}\left[\rho_{\mathrm{A}}^{\alpha}, \rho_{\mathrm{B}}^{\alpha} ; \mathbf{r}\right]
\end{aligned}
$$

where $v_{\text {ne }}(\mathbf{r})$ and $v_{\mathrm{J}}\left[\rho_{\mathrm{AB}} ; \mathbf{r}\right]$ are unchanged from Eq. (5), $v_{\mathrm{xc}}\left[\left(\rho_{\mathrm{A}}^{\alpha}+\rho_{\mathrm{B}}^{\alpha}\right),\left(\rho_{\mathrm{A}}^{\beta}+\rho_{\mathrm{B}}^{\beta}\right) ; \mathbf{r}\right]$ is the usual open-shell exchangecorrelation potential for the total system, ${ }^{8}$ and the NAKP is discussed below.
The kinetic energy functional is separable into two different spin contributions ${ }^{8}$

$$
T_{s}\left[\rho_{\mu}^{\alpha}, \rho_{\mu}^{\beta}\right]=T_{s}\left[\rho_{\mu}^{\alpha}, 0\right]+T_{s}\left[0, \rho_{\mu}^{\beta}\right]
$$

where

$$
T_{s}\left[\rho_{\mu}^{\alpha}, 0\right]=\sum_{i=1}^{N_{\mu}^{\alpha}}\left\langle\phi_{i}^{\mu, \alpha}\left|-\frac{1}{2} \nabla^{2}\right| \phi_{i}^{\mu, \alpha}\right\rangle
$$

and likewise for $T_{s}\left[0, \rho^{\beta}\right]$. Therefore, the NAKP depends only on spin densities corresponding to the same spin, such that

$$
\begin{aligned}
& v_{\text {nad }}\left[\rho_{\mathrm{A}}^{\alpha}, \rho_{\mathrm{B}}^{\alpha} ; \mathbf{r}\right]=\left.\frac{\delta T_{s}\left[\rho^{\alpha}, 0\right]}{\delta \rho^{\alpha}}\right|_{\rho^{\alpha}=\rho_{\mathrm{A}}^{\alpha}+\rho_{\mathrm{B}}^{\alpha}}(\mathbf{r})-\left.\frac{\delta T_{s}\left[\rho^{\alpha}, 0\right]}{\delta \rho^{\alpha}}\right|_{\rho^{\alpha}=\rho_{\mathrm{A}}^{\alpha}}(\mathbf{r}), \\
& v_{\text {nad }}\left[\rho_{\mathrm{A}}^{\beta}, \rho_{\mathrm{B}}^{\beta} ; \mathbf{r}\right]=\left.\frac{\delta T_{s}\left[0, \rho^{\beta}\right]}{\delta \rho^{\beta}}\right|_{\rho^{\beta}=\rho_{\mathrm{A}}^{\beta}+\rho_{\mathrm{B}}^{\beta}}(\mathbf{r})-\left.\frac{\delta T_{s}\left[0, \rho^{\beta}\right]}{\delta \rho^{\beta}}\right|_{\rho^{\beta}=\rho_{\mathrm{A}}^{\beta}}(\mathbf{r}) .
\end{aligned}
$$

The ZMP extrapolation is used to calculate the $\mathrm{KS}$ spin orbitals $\left\{\phi_{i}^{\mathrm{AB}, \nu}\right\}$ and eigenvalues $\left\{\epsilon_{i}^{\mathrm{AB}, \nu}\right\}$ for the full system, exactly as is described in the text, except that the total spin density is employed instead of the total electron density. Finally, our exact expression for the NAKP for open-shell systems is modified from Eq. (23) as follows: 


$$
\begin{aligned}
v_{\text {nad }}\left[\rho_{\mathrm{A}}^{\nu}, \rho_{\mathrm{B}}^{\nu} ; \mathbf{r}\right]= & \frac{1}{\rho_{\mathrm{AB}}^{\nu}(\mathbf{r})} \sum_{i}^{N_{\mathrm{A}}^{\nu}+N_{\mathrm{B}}^{\nu}}\left(-\frac{1}{2} \phi_{i}^{\mathrm{AB}, \nu}(\mathbf{r}) \nabla^{2} \phi_{i}^{\mathrm{AB}, \nu}(\mathbf{r})-\epsilon_{i}^{\mathrm{AB}, \nu} \phi_{i}^{\mathrm{AB}, \nu}(\mathbf{r})^{2}\right) \\
& -\frac{1}{\rho_{\mathrm{A}}^{\nu}(\mathbf{r})} \sum_{i}^{N_{\mathrm{A}}^{\nu}}\left(-\frac{1}{2} \phi_{i}^{\mathrm{A}, \nu}(\mathbf{r}) \nabla^{2} \phi_{i}^{\mathrm{A}, \nu}(\mathbf{r})-\epsilon_{i}^{\mathrm{A}, \nu} \phi_{i}^{\mathrm{A}, \nu}(\mathbf{r})^{2}\right) .
\end{aligned}
$$

The TF approximation for the non-additive kinetic energy in an open-shell calculation is

$$
T_{\mathrm{TF}}^{\mathrm{nad}}\left[\rho_{\mathrm{A}}^{\nu}, \rho_{\mathrm{B}}^{\nu}\right]=2^{2 / 3} C_{\mathrm{TF}} \int\left(\rho_{\mathrm{AB}}^{\nu 5 / 3}(\mathbf{r})-\rho_{\mathrm{A}}^{\nu 5 / 3}(\mathbf{r})-\rho_{\mathrm{B}}^{\nu 5 / 3}(\mathbf{r})\right) d \mathbf{r}
$$

and corresponding result for the TFvW functional is

$$
T_{\mathrm{TFvW}}^{\mathrm{nad}}\left[\rho_{\mathrm{A}}^{\nu}, \rho_{\mathrm{B}}^{\nu}\right]=T_{\mathrm{TF}}^{\mathrm{nad}}\left[\rho_{\mathrm{A}}^{\nu}, \rho_{\mathrm{B}}^{\nu}\right]+\frac{1}{72} \int\left(\frac{\left|\nabla \rho_{\mathrm{AB}}^{\nu}(\mathbf{r})\right|^{2}}{\rho_{\mathrm{AB}}^{\nu}(\mathbf{r})}-\frac{\left|\nabla \rho_{\mathrm{A}}^{\nu}(\mathbf{r})\right|^{2}}{\rho_{\mathrm{A}}^{\nu}(\mathbf{r})}-\frac{\left|\nabla \rho_{\mathrm{B}}^{\nu}(\mathbf{r})\right|^{2}}{\rho_{\mathrm{B}}^{\nu}(\mathbf{r})}\right) d \mathbf{r} .
$$

${ }^{1}$ P. Cortona, Phys. Rev. B 44, 8454 (1991).

${ }^{2}$ T. A. Wesolowski and A. Warshel, J. Phys. Chem. 97, 8050 (1993).

${ }^{3}$ N. Govind, Y. A. Yang, A. J. R. da Silva, and E. A. Carter, Chem. Phys. Lett. 295, 129 (1998)

${ }^{4}$ T. A. Wesołowski, in Computational Chemistry: Reviews of Current Trends, edited by J. Leszczynski (World Scientific, Singapore, 2006), Vol. 10 , pp. $1-82$.

${ }^{5}$ T. Klüner, N. Govind, Y. A. Yang, and E. A. Carter, J. Chem. Phys. 116, 42 (2002).

${ }^{6}$ G. Hong, M. Strajbl, T. A. Wesolowski, and A. Warshel, J. Comput. Chem. 21, 1554 (2000).

${ }^{7}$ M. Hodak, W. Lu, and J. Bernholc, J. Chem. Phys. 128, 014101 (2008).

${ }^{8}$ R. G. Parr and W. Yang, Density-Functional Theory of Atoms and Molecules (Oxford University Press, New York, 1989).

${ }^{9}$ A. W. Götz, S. M. Beyhan, and L. Visscher, J. Chem. Theory Comput. 5, 3161 (2009).

${ }^{10}$ M. Iannuzzi, B. Kirchner, and J. Hutter, Chem. Phys. Lett. 421, 16 (2006).

${ }^{11}$ T. A. Wesolowski, J. Chem. Phys. 106, 8516 (1997).

${ }^{12}$ A. Lembarki and H. Chermette, Phys. Rev. A 50, 5328 (1994).

${ }^{13}$ V. V. Karasiev, S. B. Trickey, and F. E. Harris, J. Comput.-Aided Mater. Des. 13, 111 (2006).

${ }^{14}$ F. Tran and T. A. Wesolowski, Int. J. Quantum Chem. 89, 441 (2002).

${ }^{15}$ Y. A. Wang, N. Govind, and E. A. Carter, Phys. Rev. B 60, 16350 (1999).

${ }^{16}$ G. K.-L. Chan, A. J. Cohen, and N. C. Handy, J. Chem. Phys. 114, 631 (2001).

${ }^{17}$ C. R. Jacob and L. Visscher, J. Phys. Chem. 128, 155102 (2008).

${ }^{18}$ S. M. Beyhan, A. W. Götz, C. R. Jacob, and L. Visscher, J. Chem. Phys. 132, 044114 (2010).

${ }^{19}$ L. H. Thomas, Proc. Cambridge Philos. Soc. 23, 542 (1927).

${ }^{20}$ E. Fermi, Z. Phys. 48, 73 (1928).

${ }^{21}$ C. F. von Weizsäcker, Z. Phys. 96, 431 (1935).

${ }^{22}$ J. P. Perdew, J. Chevary, S. Vosko, K. A. Jackson, M. R. Pederson, D. Singh, and C. Fiolhais, Phys. Rev. B 46, 6671 (1992).

${ }^{23}$ A. D. Becke, J. Chem. Phys. 84, 4524 (1986).

${ }^{24}$ R. A. King and N. C. Handy, Mol. Phys. 99, 1005 (2001).

${ }^{25}$ J. A. Alonso and L. A. Girifalco, Phys. Rev. B 17, 3735 (1978).

${ }^{26}$ P. García-González, J. E. Alvarellos, and E. Chacón, Phys. Rev. B 53, 9509 (1996).

${ }^{27}$ S. Fux, K. Kiewish, C. R. Jacob, J. Neugebauer, and M. Reiher, Chem. Phys. Lett. 461, 353 (2008).

${ }^{28}$ M. Levy, Proc. Natl. Acad. Sci. U.S.A. 76, 6062 (1979).
${ }^{29}$ Q. S. Zhao and R. G. Parr, Phys. Rev. A 46, 2337 (1992).

${ }^{30}$ Q. S. Zhao and R. G. Parr, J. Chem. Phys. 98, 543 (1993).

${ }^{31}$ Q. S. Zhao, R. C. Morrison, and R. G. Parr, Phys. Rev. A 50, 2138 (1994).

${ }^{32}$ R. A. King and N. C. Handy, Phys. Chem. Chem. Phys. 2, 5049 (2000).

${ }^{33}$ C. R. Jacob, S. M. Beyhan, and L. Visscher, J. Chem. Phys. 126, 234116 (2007).

${ }^{34}$ P. Elliott, M. H. Cohen, A. Wasserman, and K. Burke, J. Chem. Phys. 5, 827 (2009).

${ }^{35}$ S. Fux, C. R. Jacob, J. Neugebauer, L. Visscher, and M. Reiher, J. Chem. Phys. 132, 164101 (2010).

${ }^{36}$ O. Roncero, M. P. de Lara-Castells, P. Villarreal, F. Flores, J. Ortega, M. Paniagua, and A. Aguado, J. Chem. Phys. 129, 184104 (2008).

${ }^{37}$ O. Roncero, A. Zanchet, P. Villarreal, and A. Aguado, J. Chem. Phys. 131, 234110 (2009).

${ }^{38}$ P. Huang and E. A. Carter, J. Chem. Phys. 125, 084102 (2006).

${ }^{39}$ T. H. Dunning, J. Chem. Phys. 90, 1007 (1989).

${ }^{40}$ J. A. Pople, P. M. W. Gill, and N. C. Handy, Int. J. Quantum Chem. 56, 303 (1995).

${ }^{41}$ J. C. Slater, Phys. Rev. 81, 385 (1951).

${ }^{42}$ S. H. Vosko, L. Wilk, and M. Nusair, Can. J. Phys. 58, 1200 (1980).

${ }^{43}$ B. N. Papas and H. F. Schaefer III, J. Mol. Struct.: THEOCHEM 768, 175 (2006).

${ }^{44}$ M. R. Pederson and K. A. Jackson, Phys. Rev. B 41, 7453 (1990).

${ }^{45}$ W. Yang, Phys. Rev. A 34, 4575 (1986).

${ }^{46}$ L.-W. Wang and M. P. Teter, Phys. Rev. B 45, 13196 (1992).

${ }^{47}$ P. García-González, J. E. Alvarellos, and E. Chacón, Phys. Rev. A 54, 1897 (1996).

${ }^{48}$ J. M. L. Lastra, J. W. Kaminski, and T. A. Wesolowski, J. Chem. Phys. 129, 074107 (2008).

${ }^{49}$ P. Pulay, Chem. Phys. Lett. 73, 393 (1980).

${ }^{50}$ D. J. Tozer and H. C. Handy, Mol. Phys. 101, 2669 (2003).

${ }^{51}$ F. A. Hamprecht, A. J. Cohen, D. J. Tozer, and N. C. Handy, J. Chem. Phys. 109, 6264 (1998).

${ }^{52}$ A. D. Boese, N. L. Doltsinis, N. C. Handy, and M. Sprik, J. Chem. Phys. 112, 1670 (2000).

${ }^{53}$ P. J. Wilson, T. J. Bradley, and D. J. Tozer, J. Chem. Phys. 115, 9233 (2001).

${ }^{54}$ G. Menconi, P. J. Wilson, and D. J. Tozer, J. Chem. Phys. 114, 3958 (2001)

${ }^{55}$ P. Huang and E. A. Carter, Annu. Rev. Phys. Chem. 59, 261 (2008).

${ }^{56}$ S. Sharifzadeh, P. Huang, and E. A. Carter, Chem. Phys. Lett. 470, 347 (2009). 